\title{
PRINCIPES HUMANITAIRES ET RÉALITÉS POLITIQUES
}

Le 25 octobre 1986, la suspension de la représentation gouvernementale de la République d'Afrique du Sud à la XXV Conférence internationale de la Croix-Rouge a mis en cause le principe de l'universalité de la présence à la Conférence des Etats Parties aux Conventions de Genève.

Il serait tentant - mais aussi facile - de ne retenir de la Conférence que cet événement lourd de signification et d'y voir la préfiguration d'un avenir sombre pour le Mouvement de la CroixRouge et du Croissant-Rouge.

Si nous voulons croire avec le Président du CICR que la situation ainsi créée ne doit pas constituer un précédent (voir également p. 339), nous devons à ce stade raison garder et, au-delà du choc émotionnel et des images blessantes, nous efforcer, en retraçant la genèse de cette Conférence "pas comme les autres», d'en dresser un premier bilan objectif. Un bilan qui n'est pas aussi bon qu'on l'eût souhaité ni aussi mauvais qu'on aurait pu le craindre.

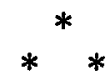

Pour la première fois depuis 1925, la Conférence internationale se tenait à Genève, ville d'Henry Dunant, berceau de la Croix-Rouge mais aussi siège européen des Nations Unies. La combinaison de ces deux éléments est un des facteurs qui ont provoqué une Conférence dont le moins qu'on puisse dire est qu'elle fut beaucoup plus délicate à gérer que les précédentes: le fait que les représentants des gouvernements, membres de Missions permanentes ou diplomates envoyés par les capitales étaient, pour la plupart, des professionnels de la diplomatie multilatérale a injecté une vigueur spéciale aux débats sur ceux des points de l'ordre du jour qui présentaient un intérêt particulier pour les gouvernements. Et pour la première fois depuis longtemps, la Conférence internationale de la Croix-Rouge s'est trouvée plongée dans un monde de réalités politiques dont elle avait été jusqu'alors, relativement "protégée».

La question de l'apartheid et les événements d'Afrique du Sud, mis en relief par les médias ces derniers mois, allaient marquer, de façon 
profonde, tout le déroulement de la Conférence. Ce qui n'était qu'un pressentiment au début de l'année 1986 allait devenir graduellement une certitude en septembre: la Conférence internationale de la CroixRouge risquait de devoir faire face à une proposition du Groupe africain d'expulser l'Afrique du Sud de la Conférence.

Certes des solutions furent envisagées, des scénarios proposés, de nombreuses démarches entreprises tant par la Commission permanente de la Croix-Rouge internationale que par le CICR ou par la Ligue des Sociétés de la Croix-Rouge et du Croissant-Rouge ainsi que par la diplomatie helvétique et par les Etats africains. Un intense dialogue s'instaura à Genève et dans les capitales. Les jours précédant la Conférence, la Genève "onusienne" vécut en contact permanent avec la Genève de la Croix-Rouge internationale, chacun s'efforçant de trouver une solution acceptable à tous, soucieux d'éviter que la Conférence soit mise à mal dans cette affaire.

Fait remarquable: avant même l'ouverture de la Conférence, l'Assemblée générale de la Ligue marqua un pas positif important dans le combat pour l'universalité du Mouvement en confirmant la légitime présence en son sein de la Société sud-africaine de la CroixRouge, qui respecte les principes d'humanité, d'impartialité et de neutralité. Cette position devait, bien heureusement, ne pas être remise en cause par la Conférence internationale.

Il allait toutefois en être autrement du gouvernement sud-africain: quoique l'Afrique du Sud soit membre de droit de la Conférence, en sa qualité de Partie aux Conventions de Genève, la délégation gouvernementale sud-africaine ne fut pas autorisée à participer aux travaux de la Conférence, à la suite d'un vote par appel nominal acquis par 159 voix contre 25 et 8 abstentions, cinquante deux délégations déclarant ne pas participer au vote (voir également p. 338).

Dans le débat qui précéda le vote, deux grandes lignes de pensée se sont dégagées:

- La première plaidait pour l'indispensable universalité du droit humanitaire, dont l'essence est de s'appliquer dans les situations conflictuelles et qui ne saurait donc, même face à une discrimination, répondre par une autre discrimination. Pour les tenants de cette approche - auxquels le CICR, pour sa part, s'identifiait il était vital de garder cet espace de dialogue que constituent, au cxur même des combats, les Conventions de Genève et le monde de la Croix-Rouge internationale.

En plus, nombreux furent ceux qui soulignèrent combien il serait dangereux de créer un précédent qui, d'exclusion en exclusion, 
risquait de conduire à la destruction définitive de l'édifice patiemment construit depuis 125 ans.

Par ailleurs, même s'il ne s'agissait pas formellement d'exclure l'Afrique du Sud mais de contester la légitimité de ses représentants, le résultat était le même, l'examen de la "représentativité" de délégations gouvernementales conduisant inévitablement à des débats politiques inextricables.

- L'autre tendance au sein de la Conférence, celle qui finalement fut majoritaire, considérait que le cas de l'apartheid était "sui generis»; pour ceux-ci, l'apartheid constitue une violation si fondamentale de ce qui fait l'essence de la dignité de l'homme, que l'on ne saurait attendre ni exiger des représentants d'un Etat africain (et de bien d'autres encore) de siéger dans la même salle que la délégation d'un régime proclamant une politique perçue comme fondée sur le racisme. Pour ceux-là, une décision de suspension du gouvernement sud-africain s'imposait au nom même de la dignité humaine que la Croix-Rouge affirme défendre dans tous les pays; elle allait dans le sens de l'Histoire et l'avenir montrerait, par cette mesure, que le Mouvement aurait enfin acquis une pleine crédibilité au sein du tiers monde; sinon ils ne sauraient, pour leur part, continuer à participer à une Conférence laissant siéger en son sein les représentants d'un tel régime.

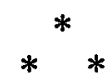

Il n'est pas douteux que cet événement marquera l'image et le souvenir de cette Conférence-là, mais il est non moins remarquable que cette décision (prise dans des conditions houleuses et sous forme d'un vote par appel nominal dont la validité fut contestée par ceux qui exigeaient le vote secret) eut une influence paradoxalement positive sur la suite des travaux. En effet, traumatisée par l'intensité des émotions, l'ardeur des arguments et la tension des procédures qui avaient abouti à cette conclusion dramatique, la Conférence éprouva le besoin de manifester son unité dans le cadre des débats sur les deux principaux thèmes de substance qui lui avaient été soumis: le respect du droit international humanitaire, d'une part, et l'approbation de nouveaux statuts pour le Mouvement international de la Croix-Rouge et du Croissant-Rouge, d'autre part.

Les premiers signes de cette volonté d'unité et de cohésion sont apparus au sein de la Commission qui traitait de la question des nouveaux statuts du Mouvement. Le texte soumis par la Ligue et le CICR constituait un consensus difficilement acquis et durement négo- 
cié, un ensemble certes généralement soutenu mais qui pouvait être remis en question si telle ou telle de ses dispositions essentielles devait se trouver altérée. Or lorsque la délégation d'une Société nationale proposa de renoncer à présenter ses propres remarques si le texte était adopté sans changement, une quarantaine de délégations de tous les continents lui apportèrent spontanément leur soutien. Cette proposition reçut l'approbation enthousiaste des délégués, consacrant ainsi la capacité de consensus et d'harmonie du Mouvement dans les moments mêmes où il était le plus menacé. L'assemblée plénière devait confirmer, dans le même esprit, cette décision.

La résolution que le CICR avait proposée sur le thème brûlant du respect du droit international humanitaire et le soutien à ses activités dans les situations de conflits armés bénéficia probablement aussi de la volonté de la Conférence de retrouver son unité vers un but commun. Dans son projet, le Comité international avait mentionné une quinzaine de conflits armés dans lesquels ses possibilités d'intervention étaient limitées, voire inexistantes, particulièrement dans le domaine de la protection des prisonniers de guerre. D'autres projets sur ces mêmes situations furent déposés par différentes délégations.

Après de longues négociations entre représentants gouvernementaux, on se mit d'accord de n'avoir qu'une seule résolution qui ne cite plus aucun pays par son nom, mais qui souligne les problèmes que rencontre le CICR dans ses activités et qui appelle toutes les Parties aux conflits mentionnés dans les rapports d'activités du CICR à respecter pleinement leurs engagements humanitaires. Fait remarquable pour ce genre de résolution: elle fut adoptée sans qu'aucune délégation ne demande le vote.

De nombreux autres points positifs sont en outre à mettre au crédit de la Conférence; on les trouvera dans les quelque 30 résolutions qu'elle a adoptées, sans qu'aucune - et cela mérite d'être souligné — n'ait dû être soumise au vote (voir aussi pp. 352-403).

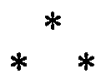

Certes l'universalité de la participation à la Conférence internationale des Etats parties aux Conventions de Genève n'a pu être maintenue. A la suite de la décision prise par la Conférence, le gouvernement sud-africain a notifié au CICR, qui n'avait pourtant pas pris part au vote incriminé, que sa délégation en Afrique du Sud devait suspendre ses activités (même si suspension ne signifie ni rupture des relations, ni résiliation de l'accord de siège). 
S'il convient de se réjouir que les autorités de la République d'Afrique du Sud aient finalement reconsidéré leur position à la fin du mois de novembre et confirmé que les délégués du CICR pouvaient rester dans le pays et y reprendre leurs activités, il n'en demeure pas moins que la suspension de la délégation gouvernementale sud-africaine à la Conférence a ébranlé tout l'édifice fondé sur l'universalité des Principes fondamentaux de la Croix-Rouge et du droit humanitaire.

Pour ceux-là, si unanimement opposés qu'ils soient au système de l'apartheid, la suspension de la délégation gouvernementale sud-africaine constitue une grave remise en cause du sens même d'une telle Conférence, qui est de permettre le dialogue avec tous les gouvernements liés par les Conventions de Genève, même - et surtout quand leur opposition est violente. N'oublions pas que des gouvernements en guerre étaient présents sur les mêmes bancs.

Pour d'autres, par contre, le fait que la Conférence internationale ait su traiter ce cas "sui generis" de l'Afrique du Sud en maintenant la Croix-Rouge sud-africaine en son sein tout en suspendant les représentants gouvernementaux du même pays, marque l'entrée définitive de la Conférence dans le monde des réalités objectives internationales et constitue un pas dans le sens d'une défense plus active de la dignité humaine.

Ainsi, pour les uns, échec de l'universalité; pour les autres, triomphe des droits de l'homme. Mais, après tout, était-il si surprenant qu'un jour "l'humanitaire» vint de cette manière buter sur "le politique" quand la Conférence internationale est le reflet d'une société mondiale en crise, sujette aux chocs constants des systèmes de valeurs également universelles? La question mérite une réflexion approfondie.

Il importe maintenant de tirer les leçons, de façon positive, des événements qui ont secoué la $X X V^{e}$ Conférence internationale de la Croix-Rouge dont le bilan est loin d'être négatif. Et c'est le Mouvement tout entier qui doit le faire dans un esprit de dialogue et avec le souci de maintenir ce qui fait sa force: son unité.

La Revue 